\title{
Impact of Age at Childbirth on Maternal Mental Health among Premenopausal Women: The 2010-2012 Korean National Health and Nutrition Examination Survey
}

\author{
Soo Hyun Joo', Sheng-Min Wang ${ }^{2}$, Jo-Eun Jeong ${ }^{3}$, Chang Tae Hahn ${ }^{3}$, and Tae-Suk Kim ${ }^{1 凶}$ \\ 1'Department of Psychiatry, Seoul St. Mary's Hospital, College of Medicine, The Catholic University of Korea, Seoul, Republic of Korea \\ 2Department of Psychiatry, Catholic Center for Brain Health, Yeouido St. Mary's Hospital, College of Medicine, The Catholic University of Korea, \\ Seoul, Republic of Korea \\ ${ }^{3}$ Department of Psychiatry, Daejeon St. Mary's Hospital, College of Medicine, The Catholic University of Korea, Daejeon, Republic of Korea
}

\begin{abstract}
Objective No reports have investigated the influence of age at first or last childbirth on maternal mental health. The aim of this study was to determine whether there is an association between age at first or last childbirth and the mental health of premenopausal women.

Methods The data used in this study were collected from the 2010 to 2012 Korea National Health and Nutrition Examination Surveys. A total of 3,370 premenopausal women were considered. The association between childbirth age and maternal mental health factors, including stress, depressed mood, and suicidal ideations were analyzed using multiple logistic regression analyses adjusted for confounding factors.

Results After adjusting confounding factors, younger maternal age at first childbirth was found to be associated with an increase in the prevalence of maternal depressed mood and suicidal ideations. Also, older maternal age at last childbirth was related to an increase in maternal stress, depressed mood and suicidal ideations.

Conclusion Both younger first childbirth and older last childbirth maternal age may be risk factors for poor outcomes of premenopausal women's mental health. These data support the need for comprehensive mental health assessment for premenopausal women who either gave birth at an age too young or too old.

Psychiatry Investig 2019;16(9):679-685
\end{abstract}

Key Words Maternal age, Mental health, Depression, Suicide, Childbirth.

\section{INTRODUCTION}

Worldwide, epidemiologic studies have suggested that an increased risk of major depressive disorder (MDD) exists in women in comparison with in men. In the United States, the National Comorbidity Survey found that the prevalence of MDD is nearly two-fold higher in women between the ages of 15 and 54 years versus in men. ${ }^{1}$ In the European Outcome of Depression International Network study, the prevalence of MDD was $10.05 \%$ [95\% confidence interval (CI), 7.80-12.85] for women versus $6.61 \%$ (95\% CI, 4.92-8.83) for men. ${ }^{2}$ This

\footnotetext{
Received: March 8, 2019 Revised: May 24, 2019

Accepted: July 9, 2019

$\triangle$ Correspondence: Tae-Suk Kim, MD, PhD

Department of Psychiatry, Seoul St. Mary's Hospital, College of Medicine, The Catholic University of Korea, 222 Banpo-daero, Seocho-gu, Seoul 06591, Republic of Korea

Tel: +82-2-2258-6085, Fax: +82-2-594-3870, E-mail: bluenote@catholic.ac.kr

(c) This is an Open Access article distributed under the terms of the Creative Commons Attribution Non-Commercial License (https://creativecommons.org/licenses/bync/4.0) which permits unrestricted non-commercial use, distribution, and reproduction in any medium, provided the original work is properly cited.
}

phenomenon was also identified in other locations such as Canada, Puerto Rico, Lebanon, Taiwan, New Zealand, and South Korea. ${ }^{3,4}$ Because depression itself is known to be closely associated with suicidal behavior including suicidal ideations, plans, or attempts, suicidal behavior is also higher in women than in men..$^{5-8}$ This phenomenon has been mainly explained by the association of female-specific hormones with depression. In particular, reproductive events and menopause are associated with depression because abrupt sex hormonal changes in these periods could influence the neurochemical pathways linked with depression. ${ }^{9,10}$ However, several psychosocial stressors may also play important roles in the development of depressive symptoms. Specifically, not only is childbirth itself a stressful condition for women; the associated economic hardship, burden of parenting, and medical complications represent additional childbirth-associated stressors. ${ }^{11,12}$

Most countries participating in the Organization for Economic Cooperation and Development (OECD) experienced a two- to four-year increase in the mean age of women giving 
birth, with an average increase from 27.5 years to 30.1 years between 1970 and $2014 .{ }^{13}$ At the same time, adolescent fertility rates remain high in low- and middle-income countries. ${ }^{13,14}$ South Korea has experienced an increase in the mean age of all women giving birth from 30.22 years in 2005 to 32.04 years in 2014. The average maternal age at first childbirth reached about 31 years as of 2014, with $21.6 \%$ of these mothers giving birth over the age of 35 years. ${ }^{15}$ Given this demographic shift, we are interested in the effect of advanced or delayed maternal age at the time of childbirth on maternal mental health.

A few studies to date have assessed the association between maternal age at childbirth and maternal mental health. ${ }^{16-19}$ However, to the best of our knowledge, there are no reports that have investigated the influence of age at the time of first or last childbirth on maternal mental health (e.g., in terms of stress, depressed mood, and suicidal ideations). Thus, the objective of the present study was to determine whether there is an association between the age at first or last childbirth and mental health in premenopausal women. We hypothesized that younger age at first childbirth or older age at last childbirth negatively impact maternal mental health.

\section{METHODS}

\section{Survey overview}

This study was conducted based on the data from the fifth Korea National Health and Nutrition Examination Survey (KNHANES V; 2010-2012), a series of nationwide crosssectional studies aimed at summarizing the health of a nationally representative, non-institutionalized sample of Korean residents. Subjects were recruited using a multi-stage clustered probability design. From approximately 200,000 primary sampling units (PSUs) defined by geographical regions within the country, the final PSUs for the actual survey were extracted. Sample weights were used to calculate all of the statistics in this survey. To ensure that the sample participants were representative of the Korean population, sample weights were created taking into account survey non-response, complex survey design, and post-stratification. Trained interviewers employed structured questionnaires to obtain information about sociodemographic, health, and lifestyle factors; the use of medical services; and female reproductive factors. All of the participants in this survey signed an informed consent form prior to their inclusion.

\section{Subjects}

Figure 1 shows the number of participants enrolled in the analysis in our study. Among all of the individuals who participated in the KNHANES V survey $(n=25,534)$, the current study included female subjects aged 19 years of age or older. 19-year-old, the adult standard of the civil law of South Korea, is also applied to the National Health and Nutrition Survey. Subjects with no pregnancy history, postmenopausal women and those with missing data such as data regarding reproductive and mental health factors were excluded. We excluded 4,370 postmenopausal women because postmenopausal women experience a significant decrease in estrogen production, which might be associated with depressive symptoms. ${ }^{20} \mathrm{~A}$ total of 3,370 premenopausal women were included in the final analysis.

\section{Measures}

Subjects' heights and weights were measured using standard procedures. Body mass index (BMI) was calculated using the following formula: $\mathrm{BMI}=$ weight $(\mathrm{kg}) \div$ height $\left(\mathrm{m}^{2}\right)$. Self-report questionnaires were used to determine smoking status; alcohol consumption; exercise; education; income; residential area; and various reproductive factors such as number of births, history of menstruation, age at childbirth, and age at last childbirth. A subject was considered to be a current smoker if she was currently smoking and had smoked at least 100 cigarettes in their lifetime. The amount of alcohol consumed (g/day) was calculated using the average number of alcoholic beverages ingested and the frequency of consumption. Risky drinking was defined as ingesting more than five alcoholic beverages on one occasion or drinking alcohol more than two days per week for women. Regular exercise was classified as either moderate or severe. Moderate exercise was defined as sessions lasting longer than 20 minutes performed at least three times per week, while severe exercise was defined as sessions lasting longer than 30 minutes performed at least five times per week. Household income was divided into quartiles and was adjusted according to the number of family members. Subsequently, income status was stratified into four quartiles; the percentage of subjects belonging to the lowest quartile group is presented in the Table 1. Geographical regions were classified as either rural or urban.

Mental health surveys included in the KNHANES V were

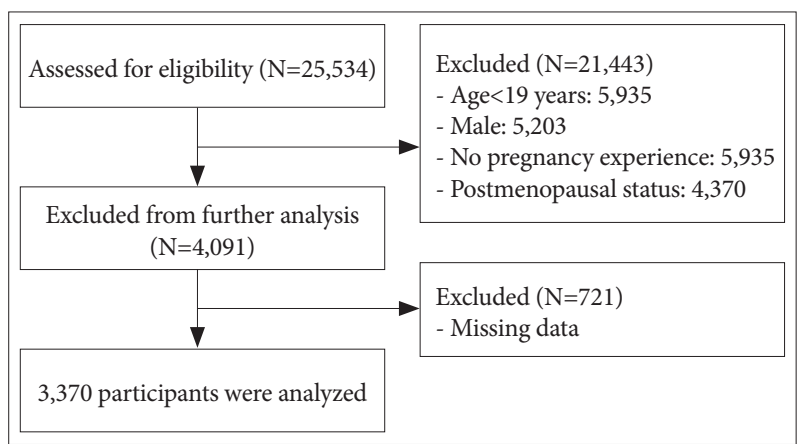

Figure 1. Flow diagram of the number of participants enrolled in this study. 
provided to all participants. Three dimensions of mental health were assessed: stress, depressed mood, and suicidal ideations. Participants answered that they "rarely feel any stress," "feel stressed a little bit," "feel stressed a lot," or "feel stressed very much" when asked that how much stress they usually feel. We defined the responses of "feel stressed a lot," or "feel stressed very much" as representing stress. To assess depressed mood, participants answered "yes" or "no" when asked whether "they have you ever experienced depressed mood for two or more continuous weeks during the last one year." Suicidal ideations were assessed with a "yes" or "no" response to the question "Have you ever had thoughts about suicide during the last one year?"

\section{Ethical issues}

The Institutional Review Board at the Korea Centers for Disease Control and Prevention approved the study protocol (nos. 2010-02CON-21-C, 2011-02CON-06-C, 2012-01EXP01-2C), and all participants signed informed consent forms prior to inclusion.

\section{Statistical analysis}

Demographic data are presented in the format of mean \pm standard error (SE) for continuous variables or percentage for categorical variables. Odds ratios (ORs) and CIs were used to identify associations between mental health and childbirth age. Selected covariates potentially affecting de-

Table 1. Sociodemographic characteristics and mental health status among pre-menopausal women

\begin{tabular}{lc}
\hline & All subjects (N=3,370) \\
\hline Age (years) & $40.4(0.2)$ \\
BMI $\left(\mathrm{kg} / \mathrm{m}^{2}\right)$ & $23.2(0.1)$ \\
Current smoking (N, \%) & $145(4.3)$ \\
Alcohol consumption (>5 g/day, N, \%) & $233(6.9)$ \\
Regular exercise (N, \%) & $590(17.5)$ \\
Lowest income quartile (N, \%) & $276(8.2)$ \\
Above high school education (N, \%) & $2939(87.2)$ \\
Spouse (N, \%) & $3131(92.9)$ \\
Region (urban area, N, \%) & $2564(76.1)$ \\
Number of births & $2.0(0.0)$ \\
Age at first childbirth, range & $26.6(0.1), 15-43$ \\
Age at last childbirth, range & $29.8(0.1), 17-49$ \\
Stress (N, \%) & $1011(30.0)$ \\
Depressed mood (N, \%) & $495(14.7)$ \\
Suicidal ideations (N, \%) & $475(14.1)$ \\
\hline
\end{tabular}

Data are presented in the format of mean (SE) for continuous variables or number (percentage) for categorical variables. BMI: body mass index pression or suicide risk such as age, lifestyle factors (e.g., smoking, alcohol consumption, regular exercise, BMI, education, income level, and marital status), and reproductive factors (e.g., number of births, age at first and age at last childbirth) were then analyzed. Model 1 was adjusted for age. Model 2 was adjusted for age, lifestyle factors as noted above, and number of births. Model 3 was adjusted for age at first or last childbirth. All statistical analyses were performed using SAS version 9.2 (SAS Institute Inc., Cary, NC, USA) to reflect the complex sampling design and weights inherent to the KNHANES and to provide nationally representative estimates. A p-value of $<0.05$ was considered indicative of statistical significance.

\section{RESULTS}

The sociodemographic characteristics and mental health status of the participants of this study are shown in Table 1. The mean age of the participants was $40.4 \pm 0.2$ years. The mean age at first childbirth was $26.6 \pm 0.1$ years, while the mean age at last childbirth was $29.8 \pm 0.1$ years. The average number of births was two. The proportion of participants that reported moderate to severe stress was 30.0\%. Depressed mood for two or more continuous weeks was reported by $14.7 \%$ of participants and suicidal ideations were reported by $14.1 \%$ of participants, respectively.

Figure 2 presented the results of percentage of premenopausal women with stress, depressed mood or suicidal ideations according to maternal age at first or last childbirth. Women who gave births their first child under the age of 20 experienced more stress, depressed mood, and suicidal ideations. Of these three mental health indicators, depressed mood and suicidal ideations were statistically significant. On the other hand, women who gave birth to their last child over the age of 40 years experienced a higher prevalence of stress, depressed mood, and suicidal ideations. However, none of these three mental health indicators were presented in a statistically significant manner.

Table 2 presents the results of the three models of multivariate logistic regressions analyzing the relationship between maternal childbirth age and mental health after adjusting the known risk factor of mental health. In model 1, age adjustment was conducted to minimize possible bias from the participants' age at the survey. The results showed that rate of stress, depressed mood, suicidal ideation did not have significant correlation with age at first or last childbirth. Thereafter, in addition to age, life styles factors (smoking, alcohol consumption, exercise, body mass index, education, income level, and marital status) and number of births were adjusted as cofactors in model 2 . The results again did not 


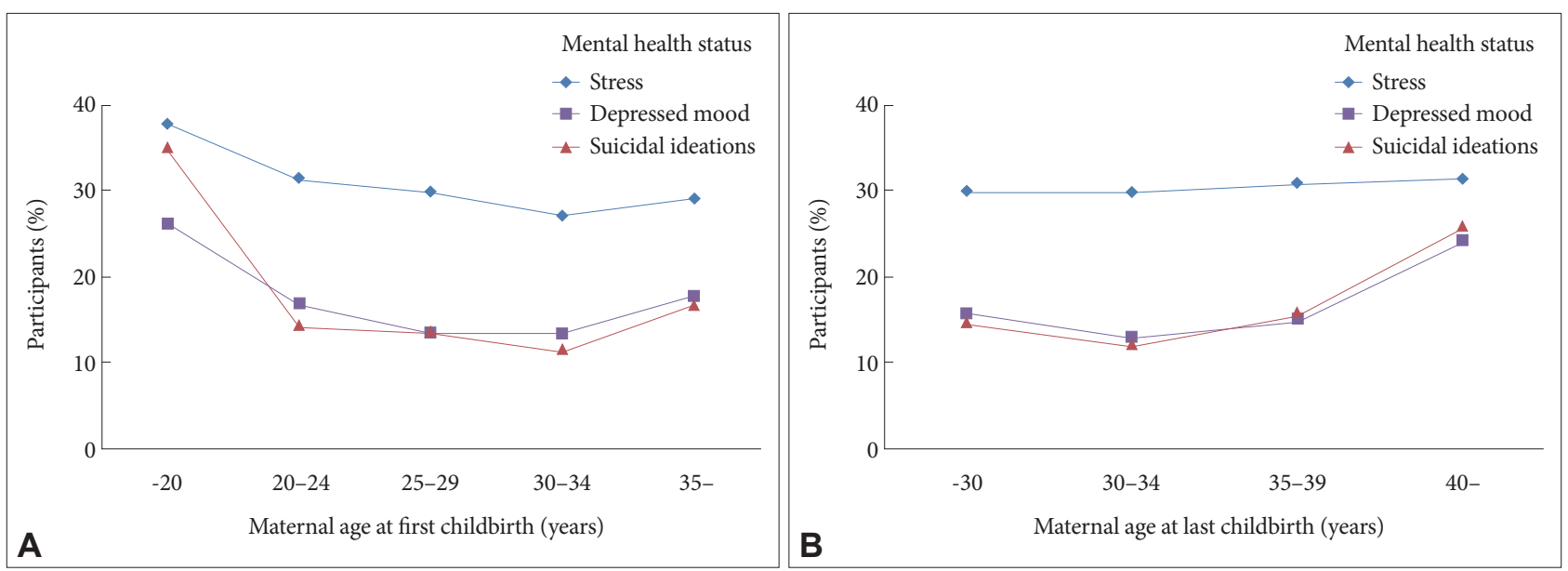

Figure 2. Mental health status and maternal age at first or last childbirth. A: Percentage of pre-menopausal women with stress, depressed mood or suicidal ideations according to maternal age at first childbirth. The p-values of stress, depressed mood, and suicidal ideations were $0.51,0.05$, and $<0.01$, respectively, B: Percentage of premenopausal women with stress, depressed mood or suicidal ideations according to maternal age at last childbirth. The $p$-values of stress, depressed mood, and suicidal ideations were $0.98,0.15$, and 0.06 , respectively.

show any significant findings. Age at first and the last childbirth could be a cofounding factor for each other (i.e. a woman with higher stress, depressed mood, and suicidal ideation might had her first child delivery before 20 and had her last child delivery after 40). Thus, in order to investigate age of first or last childbirth in mental health independently, model 3 adjusted the maternal age of the childbirth in addition to the cofactors adjusted in model 2. For example, the maternal age at last childbirth was adjusted when studying the relationship between maternal age at first childbirth and maternal mental health, whereas the maternal age at first childbirth was adjusted when investigating the relationship between maternal age at last childbirth and maternal mental health. The results showed that older maternal age at first childbirth was negatively associated with depressed mood ( $\mathrm{OR}=0.93,95 \% \mathrm{CI}=0.88-0.98$ ), while suicidal ideations $(\mathrm{OR}=0.94,95 \% \mathrm{CI}=0.89-0.99)$ after adjusting for age, lifestyle and reproductive factors, and age at last childbirth (Model 3). Older maternal age at last childbirth was positively associated with stress $(\mathrm{OR}=1.05,95 \% \mathrm{CI}=1.01-1.09)$, depressed mood $(\mathrm{OR}=1.07,95 \% \mathrm{CI}=1.02-1.12)$, and suicidal ideations ( $\mathrm{OR}=1.07,95 \% \mathrm{CI}=1.02-1.13)$ after adjusting for age, lifestyle and reproductive factors, and age at first childbirth (Model 3).

\section{DISCUSSION}

The principal findings of the present study are that younger age at the time of first childbirth is associated with an increase in maternal depressed mood and suicidal ideations and that older age at the time of last childbirth is associated with an increase in maternal stress, depressed mood and suicidal ideations in pre-menopausal women, respectively. This
Table 2. Effects of childbirth age on mental health status among pre-menopausal women

\begin{tabular}{|c|c|c|}
\hline & Mental health status & OR (95\% CI) \\
\hline \multicolumn{3}{|c|}{ Age at first childbirth } \\
\hline \multirow[t]{3}{*}{ Model 1} & Stress & $0.98(0.96-1.00)$ \\
\hline & Depressed mood & $0.98(0.95-1.01)$ \\
\hline & Suicidal ideations & $0.98(0.95-1.01)$ \\
\hline \multirow[t]{3}{*}{ Model 2} & Stress & $1.00(0.98-1.02)$ \\
\hline & Depressed mood & $0.99(0.96-1.02)$ \\
\hline & Suicidal ideations & $1.00(0.97-1.03)$ \\
\hline \multirow[t]{3}{*}{ Model 3} & Stress & $0.96(0.92-1.00)$ \\
\hline & Depressed mood & $0.93(0.88-0.98)^{*}$ \\
\hline & Suicidal ideations & $0.94(0.89-0.99)^{*}$ \\
\hline \multicolumn{3}{|c|}{ Age at last childbirth } \\
\hline \multirow[t]{3}{*}{ Model 1} & Stress & $1.01(0.99-1.03)$ \\
\hline & Depressed mood & $0.98(0.95-1.00)$ \\
\hline & Suicidal ideations & $0.99(0.97-1.02)$ \\
\hline \multirow[t]{3}{*}{ Model 2} & Stress & $1.02(1.00-1.04)$ \\
\hline & Depressed mood & $1.01(0.99-1.04)$ \\
\hline & Suicidal ideations & $1.03(1.00-1.06)$ \\
\hline \multirow[t]{3}{*}{ Model 3} & Stress & $1.05(1.01-1.09)^{*}$ \\
\hline & Depressed mood & $1.07(1.02-1.12)^{*}$ \\
\hline & Suicidal ideations & $1.07(1.02-1.13)^{*}$ \\
\hline
\end{tabular}

Results are expressed in the format of OR (95\% CI). Model 1: Adjustment for age. Model 2: Model 1 plus adjustment for lifestyle factors (e.g., smoking, alcohol consumption, exercise, body mass index, education, income level, and marital status) and number of births. Model 3: Model 2 plus adjustment for age at first or last childbirth; The maternal age at last childbirth was adjusted when studying the relationship between maternal age at first childbirth and maternal mental health, whereas the maternal age at first childbirth was adjusted when investigating the relationship between maternal age at last childbirth and maternal mental health. *statistically significant values 
study suggests that maternal childbirth age may have a direct or indirect negative effect on the mental health of pre-menopausal women.

There is a scarcity of data concerning the association between age at childbirth and maternal mental health. Moreover, the ages defining a younger or an older mother are inconsistent, making it difficult to compare the studies that do exist to one another. Therefore, defining the unique characteristics that impact psychosocial stress and subsequently mental health in the case of early or advanced maternal age remains challenging. Notably, there are a few studies targeting teenage mothers. The longitudinal consequences of adolescent pregnancy in terms of mental health difficulties, including depression, suicide attempt, and alcohol or drug abuse, have been reported. ${ }^{16,21}$ These issues were associated with young mothers with lower educational and occupational attainment, increased risk of poverty and welfare dependence, larger family size, and increased risk of divorce. ${ }^{21,22}$ Women who gave birth at a younger age were less likely to maintain a good academic status or career, which can lead to low self-esteem, low satisfaction, and economical difficulties. This increases the likelihood of mental health problem for women who gave birth to their first child at a younger age. In our study, women who experienced first childbirth under the age of 19 had lower education level, income and spouse while the number of birth was higher than women who experienced first childbirth over the age of 20. However, after adjusting these factors, younger age at the time of first childbirth is associated with negative mental health of women.

There was a significant association between older maternal age at last childbirth and maternal mental health indicators such as stress, depressed mood, and suicidal ideations in this study. Older mothers are more likely to experience negative gestational and labor events or miscarriage and that such incidences are linearly correlated with age. ${ }^{19,23}$ These reproductive difficulties are also likely to negatively affect maternal mental health. Boivin et al. ${ }^{19}$ also found that mothers in older age groups (i.e., those older than 38 years) reported significantly more depressive symptoms and fewer expressed warmth towards their partner as compared with their counterparts in the younger group (i.e., those younger than 31 years). However, another study reported that older maternal age was not related to the prevalence or timing of major depressive episodes in the first two years after birth. ${ }^{17}$ In fact, most studies have reported on the effects of maternal age on their children. These studies show that maternal childbirth age does not have any impact on child wellbeing during early and middle childhood ${ }^{19}$ and that older mothers provided more sensitivity (e.g. warmth and emotional connection) and a more structured environment to five-month old infants. ${ }^{24}$ Older mothers have typically accrued more life experiences, wisdom, financial and social resources, and a more varied coping repertoire that promotes a more responsive family environment in comparison with younger mothers. ${ }^{24}$ However, although older mothers may not have a negative impact on their children, they are more likely to feel deprivation due to delayed childbirth and parenting than their peers. In our study, women who experienced last childbirth over the age of 40 had lower income while higher number of birth than women who experienced last childbirth under the age of 39. However, after adjusting these factors, older age at the time of last childbirth is associated with negative mental health of women.

There are some limitations to present study. First, this study was based on a cross-sectional design. Further longitudinal studies will be needed to clarify the relationship between childbirth age and maternal mental health. Second, the survey only collected the participants' self-reported information, potentially compromising the objectivity of the study. Furthermore, this study survey did not use validated questionnaires for depression (including suicidal ideations) and stress. Third, we did not adjust for other physical factors of the participants such as chronic obstructive pulmonary disease, ${ }^{25}$ hormone replacement therapy, ${ }^{26}$ olfactory dysfunction, ${ }^{27}$ diabetes mellitus, ${ }^{28}$ and cancer, ${ }^{29}$ which might affect the prevalence of depressed mood or suicidal ideations. Fourth, we did not consider other psychiatric disorders such as bipolar disorder, ${ }^{6}$ alcohol use disorder, ${ }^{30}$ anxiety disorder, ${ }^{5}$ psychotic disorder, ${ }^{5}$ post-traumatic stress disorder, ${ }^{31}$ or personality disorder, ${ }^{32}$ which are also well-known risk factors of suicide and depressive disorder. Fifth, there was no information available from the KNHANES $V$ regarding family history of suicide or psychiatric disorders, which increase the risk of suicide or depressive disorder. ${ }^{33}$ Sixth, the intervals between the time of childbirth and the onset of mental health problems were various. For this reason, we cannot explain the sequential relationship between childbirth and mental health problems. Seventh, because this study analyzed the national data of Korean adult population, it is a limitation that 19 years old women who are adults of Korea are included in this study.

In spite of these several limitations, this study has important implications. First, to the best of our knowledge, this is the first study to investigate the relationship between maternal mental health and maternal age at first and last childbirth. Our study has provided important data that could increase the available knowledge about risk factors of premenopausal women's mental health. In particular, the present study could provide important information when researching women's mental health in OECD countries, where the childbirth age of women is increasing. Second, KNHANES is a large, nationally representative survey in Korea and the questionnaires were 
conducted by trained interviewers in a standardized manner, so the data presented is highly reliable.

In conclusion, this study suggests that both younger maternal age at first childbirth and older maternal age at last childbirth may be risk factors for poor outcomes of premenopausal women's mental health including depression and suicide. These data support the need for comprehensive mental health assessment in women who give birth either too young or too old. Further prospective cohort studies investigating the causal relationships between childbirth age and maternal mental health are also needed.

\section{Acknowledgments}

The authors would like to thank the Korean Center for Disease Control and Prevention, which conducted the KNHANES studies and the subjects who participated in the KNHANES.

\section{Conflicts of Interest}

The authors have no potential conflicts of interest to disclose.

\section{Author Contributions}

Conceptualization: Soo Hyun Joo, Jo-Eun Jeong, Chang Tae Hahn, TaeSuk Kim. Data curation: Soo Hyun Joo, Jo-Eun Jeong, Chang Tae Hahn. Formal analysis: Soo Hyun Joo, Tae-Suk Kim. Validation: Tae-Suk Kim. Writing_original draft: Soo Hyun Joo. Writing_-review \& editing: ShengMin Wang, Tae-Suk Kim.

\section{ORCID iDs}

$\begin{array}{ll}\text { Tae-Suk Kim } & \text { https://orcid.org/0000-0002-2488-2904 } \\ \text { Soo Hyun Joo } & \text { https://orcid.org/0000-0003-1390-3623 }\end{array}$

\section{REFERENCES}

1. Kessler RC, Berglund P, Demler O, Jin R, Koretz D, Merikangas KR, et al. The epidemiology of major depressive disorder: results from the National Comorbidity Survey Replication (NCS-R). JAMA 2003; 289:3095-3105.

2. Ayuso-Mateos JL, Vázquez-Barquero JL, Dowrick C, Lehtinen V, Dalgard OS, Casey P, et al. Depressive disorders in Europe: prevalence figures from the ODIN study. Br J Psychiatry 2001;179:308-316.

3. Weissman MM, Bland RC, Canino GJ, Faravelli C, Greenwald S, Hwu HG, et al. Cross-national epidemiology of major depression and bipolar disorder. JAMA 1996;276:293-299.

4. Ohayon MM, Hong SC. Prevalence of major depressive disorder in the general population of South Korea. J Psychiatr Res 2006;40:30-36.

5. Hirokawa S, Kawakami N, Matsumoto T, Inagaki A, Eguchi N, Tsuchiya $\mathrm{M}$, et al. Mental disorders and suicide in Japan: a nation-wide psychological autopsy case-control study. J Affect Disord 2012;140:168175 .

6. Jeon HJ, Lee JY, Lee YM, Hong JP, Won SH, Cho SJ, et al. Lifetime prevalence and correlates of suicidal ideation, plan, and single and multiple attempts in a Korean nationwide study. J Nerv Ment Dis 2010;198:643-646.

7. McGirr A, Renaud J, Séguin M, Alda M, Turecki G. Course of major depressive disorder and suicide outcome: a psychological autopsy study. J Clin Psychiatry 2008;69:966-970.

8. Nock MK, Hwang I, Sampson NA, Kessler RC. Mental disorders, comorbidity and suicidal behavior: results from the National Comorbidity Survey Replication. Mol Psychiatry 2010;15:868-876.

9. Cyranowski JM, Frank E, Young E, Shear MK. Adolescent onset of the gender difference in lifetime rates of major depression: a theoretical model. Arch Gen Psychiatry 2000;57:21-27.

10. Soares CN, Zitek B. Reproductive hormone sensitivity and risk for depression across the female life cycle: a continuum of vulnerability? J Psychiatry Neurosci 2008;33:331-343.

11. Field T, Hernandez-Reif M, Diego M. Risk factors and stress variables that differentiate depressed from nondepressed pregnant women. Infant Behav Dev 2006;29:169-174.

12. Rubertsson C, Wickberg B, Gustavsson P, Rådestad I. Depressive symptoms in early pregnancy, two months and one year postpartumprevalence and psychosocial risk factors in a national Swedish sample. Arch Womens Ment Health 2005;8:97-104.

13. OECD. Age of Mothers at Childbirth. Available at: http://www.oecd. org/els/family/database.htm. Accessed August 15, 2014.

14. Adolescent pregnancy. Available at: http://www.who.int/mediacentre/ factsheets/fs364/en/. Accessed April 8, 2016.

15. Korean National Statistical Office. 2014 Death and Cause of Death in Korea. Daejon: Korean National Statistical Office; 2015.

16. Iacobelli S, Robillard PY, Gouyon JB, Nichols M, Boukerrou M, Barau $\mathrm{G}$, et al. Longitudinal health outcome and wellbeing of mother-infant pairs after adolescent pregnancy in Reunion Island, Indian Ocean. Int J Gynaecol Obstet 2014;125:44-48.

17. McMahon CA, Boivin J, Gibson FL, Hammarberg K, Wynter K, Fisher JR. Older maternal age and major depressive episodes in the first two years after birth: findings from the Parental Age and Transition to Parenthood Australia (PATPA) study. J Affect Disord 2015;175:454-462.

18. Vicary JR, Corneal DA. A comparison of young women's psychosocial status based on age of their first childbirth. Fam Community Health 2001;24:73-84.

19. Boivin J, Rice F, Hay D, Harold G, Lewis A, van den Bree MM, et al. Associations between maternal older age, family environment and parent and child wellbeing in families using assisted reproductive techniques to conceive. Soc Sci Med 2009;68:1948-1955.

20. Tsiligianni IG, Tyrovolas S, Bountziouka V, Zeimbekis A, Gotsis E, Metallinos G, et al. Depressive symptoms in postmenopausal women: results from the MEDIS Study. Women Health 2014;54:389-401.

21. Moffitt TE, E-Risk Study Team. Teen-aged mothers in contemporary Britain. J Child Psychol Psychiatry 2002;43:727-742.

22. Furstenberg FF, Brooks-Gunn J, Morgan SP. Adolescent Mothers in Later Life. Cambridge Cambridgeshire; New York: Cambridge University Press; 1987.

23. Bewley S, Davies M, Braude P. Which career first? BMJ 2005;331:588589.

24. Bornstein MH, Putnick DL, Suwalsky JT, Gini M. Maternal chronological age, prenatal and perinatal history, social support, and parenting of infants. Child Dev 2006;77:875-892.

25. Chung JH, Han CH, Park SC, Kim CJ. Suicidal ideation and suicide attempts in chronic obstructive pulmonary disease: the Korea National Health and Nutrition Examination Survey (KNHANES IV, V) from 2007-2012. NPJ Prim Care Respir Med 2014;24:14094.

26. Lee JY, Park YK, Cho KH, Kim SM, Choi YS, Kim DH, et al. Suicidal ideation among postmenopausal women on hormone replacement therapy: The Korean National Health and Nutrition Examination Survey (KNHANES V) from 2010 to 2012. J Affect Disord 2016;189:214219.

27. Joo YH, Hwang SH, Han KD, Seo JH, Kang JM. Relationship between olfactory dysfunction and suicidal ideation: The Korea National Health and Nutrition Examination Survey. Am J Rhinol Allergy 2015;29:268-272.

28. Chung JH, Moon K, Kim do H, Min JW, Kim TH, Hwang HJ. Suicidal ideation and suicide attempts among diabetes mellitus: the Korea National Health and Nutrition Examination Survey (KNHANES IV, V) from 2007 to 2012. J Psychosom Res 2014;77:457-461.

29. Kim KH, Cho YY, Shin DW, Lee JH, Ko YJ, Park SM. Comparison of physical and mental health status between cancer survivors and the general population: a Korean population-based survey (KNHANES 
II-IV). Support Care Cancer 2013;21:3471-3481.

30. Joo SH, Wang SM, Kim TW, Seo HJ, Jeong JH, Han JH, et al. Factors associated with suicide completion: a comparison between suicide attempters and completers. Asia Pac Psychiatry 2016;8:80-86.

31. Bernal M, Haro JM, Bernert S, Brugha T, de Graaf R, Bruffaerts R, et al. Risk factors for suicidality in Europe: results from the ESEMED study. J Affect Disord 2007;101:27-34.
32. Hawton K, Saunders K, Topiwala A, Haw C. Psychiatric disorders in patients presenting to hospital following self-harm: a systematic review. J Affect Disord 2013;151:821-830.

33. Qin P, Agerbo E, Mortensen PB. Suicide risk in relation to socioeconomic, demographic, psychiatric, and familial factors: a national register-based study of all suicides in Denmark, 1981-1997. Am J Psychiatry 2003;160:765-772. 